\title{
The renal papilla is a niche for adult kidney stem cells
}

\author{
Juan A. Oliver, ${ }^{1}$ Omar Maarouf, ${ }^{1}$ Faisal H. Cheema, ${ }^{2}$ Timothy P. Martens, ${ }^{2}$ and Qais Al-Awqati ${ }^{1,3}$ \\ 1Department of Medicine, ${ }^{2}$ Department of Surgery, and ${ }^{3}$ Department of Physiology and Cellular Biophysics, Columbia University, New York, New York, USA.
}

\begin{abstract}
Many adult organs contain stem cells, which are pluripotent and are involved in organ maintenance and repair after injury. In situ, these cells often have a low cycling rate and locate in specialized regions (niches). To detect such cells in the kidney, we administered a pulse of the nucleotide bromodeoxyuridine (BrdU) to rat and mouse pups and, after a long (more than 2-month) chase, examined whether the kidney contained a population of low-cycling cells. We found that in the adult kidney, BrdU-retaining cells were very sparse except in the renal papilla, where they were numerous. During the repair phase of transient renal ischemia, these cells entered the cell cycle and the BrdU signal quickly disappeared from the papilla, despite the absence of apoptosis in this part of the kidney. In vitro isolation of renal papillary cells showed them to have a plastic phenotype that could be modulated by oxygen tension and that when injected into the renal cortex, they incorporated into the renal parenchyma. In addition, like other stem cells, papillary cells spontaneously formed spheres. Single-cell clones of these cells coexpressed mesenchymal and epithelial proteins and gave rise to myofibroblasts, cells expressing neuronal markers, and cells of uncharacterized phenotype. These data indicate that the renal papilla is a niche for adult kidney stem cells.
\end{abstract}

\section{Introduction}

Although cell division is infrequent in the adult kidney, this organ has the capacity to regenerate and repair, as illustrated by its cellular proliferation and functional recovery after ischemic tubular necrosis $(1,2)$. The origin of newly generated renal cells is largely undefined, but some cells appear to derive from division of fully differentiated cells $(3,4)$, and recent work has shown that bone marrow cells could repopulate the nephron after ischemia (5-7). In addition, by analogy with other organs, it is likely that new cells also derive from organ-specific pluripotent cells (i.e., adult renal stem cells). Organspecific stem cells were initially recognized in the hematopoietic system, skin, and intestinal epithelia, where self-renewal is manifestly apparent, but they are also present in organs that do not have rapid rates of cell turnover, such as the nervous system, prostate, liver, etc. Although the number of organs known to harbor adult stem cells continues to grow, it remains unknown whether the adult kidney possesses stem cells among its many cell types.

In searching for stem cells in the adult kidney, we reasoned that the characteristically slow cycling time of organ-specific adult stem cells (8-10) might provide a useful strategy for their recognition. Cells with slow cycling time can be distinguished by retention of a nucleotide label such as bromodeoxyuridine (BrdU), which is incorporated into the DNA of cells during DNA synthesis. If after administration of a pulse of BrdU the cells are monitored for long periods of chase, only the slowly cycling cells retain a concentration of label sufficiently high to allow their staining and thus adult organ-specific stem cells are often called "label-retaining cells" (10). Accordingly, to identify adult kidney-specific stem cells, we administered BrdU to 3-day-old rat and mice pups, an age in which, as nephrogenesis in rodents continues after birth,

Nonstandard abbreviations used: BrdU, bromodeoxyuridine; HIF-1 $\alpha$, hypoxiainducible factor- $1 \alpha$; LIF, leukemia inhibitory factor.

Conflict of interest: The authors have declared that no conflict of interest exists.

Citation for this article: J. Clin. Invest. 114:795-804 (2004)

doi:10.1172/JCI200420921. many cells in the kidney were probably dividing and thus could incorporate BrdU. After a chase period of at least 2 months, during which the multiple cell divisions required for kidney growth would have diluted the BrdU content of most cells, we analyzed the adult kidneys. We found that only the renal papilla contained an abundant population of cells that retained a strong BrdU signal. These cells are probably involved in renal repair, because although they remained in the papilla throughout the life of the animal, they proliferated and disappeared from the papilla during the repair phase of transient renal ischemia. Papillary cell proliferation after transient ischemia occurred even though the renal papilla, unlike other areas of the kidney, displayed no apoptosis after the ischemic insult. Isolation of renal papillary cells showed that in vitro the cells are multipotent and display other characteristics of adult stem cells and that when injected directly into the renal cortex, they incorporated into the kidney parenchyma. The results indicate that the renal papilla is a niche for a population of adult kidney stem cells involved in kidney maintenance and repair.

\section{Results}

BrdU-retaining cells in adult kidney. We administered BrdU to 3-dayold rat pups for a period of 3.5 days and harvested their kidneys 2 or more months later. BrdU is incorporated into the DNA during the $S$ phase of the cell cycle, but during the chase period, further DNA synthesis in the absence of ambient BrdU increasingly dilutes the label in the daughter cells. Hence, the intensity of the label in the cell nucleus is an index of the number of cell divisions that have occurred since BrdU was administered, with the brightest cells being those with the lowest number of divisions. Immunofluorescence microscopy was used to detect BrdU-labeled cells. Standard kidney sections $5 \mu \mathrm{m}$ in thickness very rarely contained any cells positive for BrdU, except in the kidney papilla, where some cells were detected (not shown), but a more detailed survey of the label-retaining cells was possible in sections $100 \mu \mathrm{m}$ in thickness. Kidneys of four independent litters of 2- to 10-monthold rats showed similar results, and representative examples are 

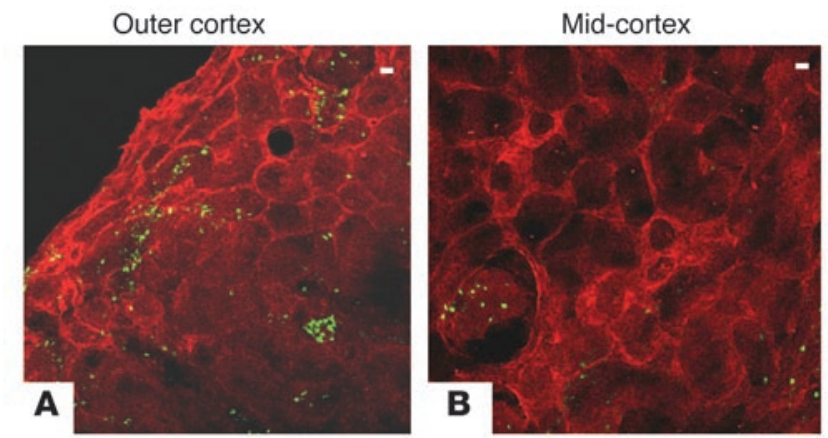

Medulla
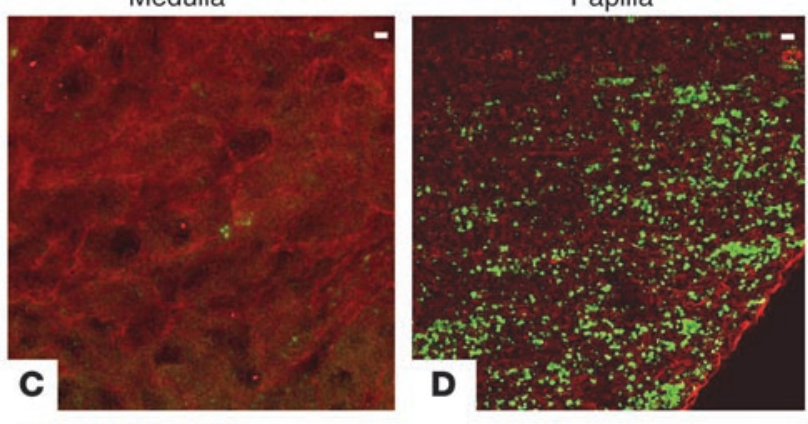

Collagen IV; BrdU

presented in Figures 1 and 2. Figure 1 shows sections of different regions of a single kidney stained for BrdU (FITC fluorescin) and collagen IV (rhodamine). The overwhelming majority of the kidney parenchyma had no cells positive for BrdU. In the renal cortex there were some scattered BrdU-positive cells, most of which were present in the subcapsular region (Figure 1A). While most glomeruli had no BrdU-positive cells (Figure 2A), some glomeruli contained a few BrdU-retaining cells (Figures $1 \mathrm{~B}$ and $2 \mathrm{~B}$ ). There were very rare BrdU-positive cells in cortical tubules. The kidney medulla contained extremely rare BrdU-positive cells (Figures $1 \mathrm{C}$ and $2 \mathrm{C})$. In marked contrast to the rest of the kidney, large numbers of BrdU-retaining cells were present in the kidney papilla (Figures 1D and 2, D-F). Figure 2E shows that the fluorescent signal emitted by the BrdU-retaining cells in the papilla was heterogeneous, with the highest intensity located in the outer part of the papilla, and Figure $2 \mathrm{~F}$ shows that in the papillary region closest to the urinary space, BrdU-retaining cells were detected even in 5- $\mu \mathrm{m}$ sections.

Detailed study of papillary BrdU-retaining cells showed that they were mostly located in the interstitium (Figure 2D, arrows) but, remarkably, some were also tubular epithelial cells (Figure 2D, arrowheads). These BrdU-retaining cells were costained with $\mathrm{ZO}-1$ antibodies, a marker for epithelial tight junctions; Figure $2 \mathrm{G}$ shows a papillary tubule with BrdU-retaining epithelial cells. Most BrdUretaining interstitial cells were surrounded by a collagen IV matrix (Figure 2H). Finally, given that the papilla is richly vascularized and adult organ-specific stem cells have been found in close association with blood vessels (11), we examined the relation of BrdUretaining cells with endothelial cells. Double immunostaining with antibody against BrdU and antibody against von Willebrand factor showed that the BrdU-retaining cells were in close association with endothelial cells but that the BrdU signal and the von Willebrand signal did not overlap (Figure 2I).

The finding that the papilla of the adult mammalian kidney harbors a population of cells that rarely divide was confirmed

\section{Figure 1}

BrdU-retaining cells in the adult kidney. Composite views $100 \mu \mathrm{m}$ in thickness of a kidney from a 2-month-old rat, obtained by confocal microscopy with 2- $u$ m optical sections. (A) Outer cortex, showing few scattered BrdU-retaining cells with an occasional cell aggregate. (B) Midcortex, showing a glomerulus with BrdU-positive cells. (C) Medulla with very rare BrdU-positive cells. (D) Papilla with numerous BrdU-retaining cells. Colored labels indicate staining with FITC fluorescin (green) and rhodamine (red) as described in Results. Scale bars: $50 \mu \mathrm{m}$.

in mice; 3 -day-old pups were given BrdU and their adult kidneys were examined. Figure 3 shows results in the kidney of a 3month-old mouse stained for BrdU (FITC fluorescin) and with an antibody against collagen IV (rhodamine). While the outer cortex (Figure 3A), like other parts of the kidney (not shown), rarely contained BrdU-retaining cells, the kidney papilla (Figure 3B) had large numbers of these cells.

Isolation of renal papillary cells. Dispersed cells from adult renal papilla were seeded on plastic or on fibronectin-coated dishes and were grown in tissue culture. Compared with plastic, fibronectin increased the rate of cellular proliferation and decreased the number of cell aggregates (see below), but no other differences were noted when the cells were seeded on this matrix. Very few cells survived isolation; flow cytometry analysis of three independent experiments showed that from the renal papillae of 6 rats, about $2 \times 10^{6}$ to $3 \times 10^{6}$ cells were viable, as defined by exclusion of propidium iodide (not shown), but seeding these cells resulted in less than $1 \times 10^{4}$ cells about 7 days after isolation. Isolation of the cells under hypertonic conditions by the addition of $500 \mathrm{mM}$ urea and $350 \mathrm{mM} \mathrm{NaCl}$ to all buffers and culture media did not increase cell yield.

For the first 2 days after isolation, and despite the fact that the cells were isolated by filtration through a $20-\mu \mathrm{m}$ filter, papillary cells formed aggregates, with $\mathrm{ZO}-1$ being detectable in the intercellular junctions (Figure 4A). To determine the frequency at which the BrdU-retaining renal papillary cells were isolated by our procedure, we cultured papillary cells from adult rats loaded with BrdU at 3 days of age followed by a 2 -month chase. After 2 days of isolation, cells adhered to the culture dish, and quantification of the BrdUpositive cells was possible. In three independent isolations each derived from 6 rats, an average of $40 \% \pm 6 \%$ of the cells were BrdUretaining cells (an example is shown in Figure 4, B and C).

Because in the kidney $(6,7)$ as well as other organs such as muscle (12), bone marrow-derived cells may differentiate into parenchymal cells and participate in organ maintenance and repair, we examined whether the isolated papillary cells contained a population of cells that originated in the bone marrow. Flow cytometry of the acutely dispersed cells from the papillae of 8 rats revealed that $3 \%$ of the cells were positive for CD 45 , indicating that a small fraction of the isolated cells were blood cells. However, less than $0.5 \%$ of the total cells were positive for CD34, CD44, or c-Kit, indicating that if hematopoietic stem cells or bone marrow mesenchymal stem cells were isolated with the renal papillary cells, their contribution to the population of the BrdU-retaining cells (about $40 \%$ of the total cells) was small. Because the kidney has a very high blood supply, it is likely that these cells represented "contamination" with intrarenal blood elements.

Shortly after attachment to the culture dish, most cells developed epithelial characteristics, with ZO-1 being prominently expressed in their tight junctions (Figure 4D); however, as we pre- 


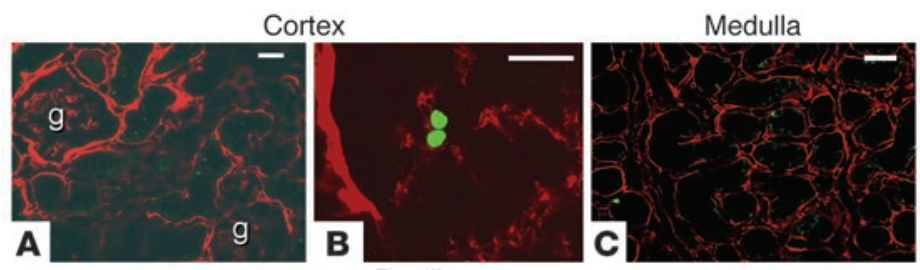

Papilla
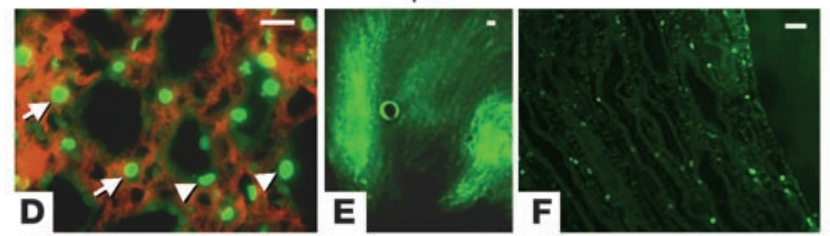

Collagen IV; BrdU

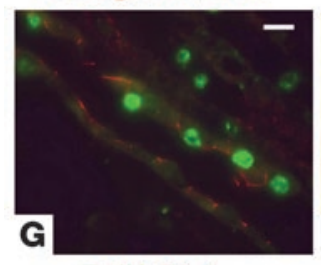

BrdU; ZO-1
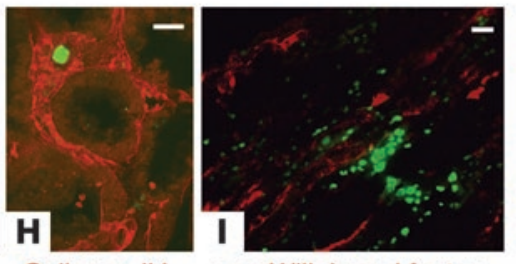

Collagen IV von Willebrand factor

\section{Figure 2}

BrdU-retaining cells in the adult kidney. (A-F) Composite views obtained by confocal microscopy with 1- $\mu \mathrm{m}$ optical sections, except where indicated otherwise (E and $\mathbf{F})$. (A) Composite view $20 \mu \mathrm{m}$ in thickness of a kidney cortex from a 3-month-old rat showing no BrdU-positive cells. g, glomerulus. (B) Composite view $10 \mu \mathrm{m}$ in thickness of a glomerulus from a 2-month-old rat with a single BrdU-retaining cell in the glomerular tuft. (C) Composite view $20 \mu \mathrm{m}$ in thickness of a kidney medulla from a 2-month-old rat with a single BrdU-retaining cell. (D) Composite view $20 \mu \mathrm{m}$ in thickness of a kidney papilla from a 2-month-old rat showing numerous BrdU-positive cells. Arrows, interstitial cells; arrowheads, tubular cells. (E) Section $100 \mu \mathrm{m}$ in thickness of a kidney papilla from a 3-month-old rat showing more fluorescent signal (i.e., $\mathrm{BrdU}$ ) in the outer parts of the papilla. (F) Section $5 \mu \mathrm{m}$ in thickness of the kidney papilla from a 5-month-old rat. (G) Composite view $20 \mu \mathrm{m}$ in thickness of kidney papillary tubules from a 4-month-old rat showing that some of the BrdU-retaining cells are tubular epithelial cells expressing ZO-1 in their tight junctions. (H) Composite view $10 \mu \mathrm{m}$ in thickness of a kidney medulla from a 3-month-old rat showing a single interstitial BrdU-retaining cell surrounded by collagen IV. (I) Composite view $20 \mu \mathrm{m}$ in thickness of a kidney papilla from a 4-month-old rat showing BrdU-retaining cells in close association with endothelial cells. Scale bars: $50 \mu \mathrm{m}$.

viously found in metanephric mesenchymal stem cells (13), many papillary cells coexpressed both epithelial and mesenchymal proteins such as $\alpha$-smooth muscle actin (Figure 4E). Under control culture conditions, within 2 weeks of seeding, all cells became spindle-shaped and expressed $\alpha$-smooth muscle actin (Figure 4F). However, when the cells were grown in $1.5 \% \mathrm{O}_{2}$, the vast majority of the cells retained the epithelial phenotype (Figure 4G). We point out that the renal papilla has low oxygen tension.

Cellular spheres. Embryonic stem cells and adult organ-specific stem cells grown in culture easily form aggregates and/or spheres (14-17), called neurospheres in the case of neuronal stem cells, and it can often be determined that these spheres derive from a single cell (17). Although renal papillary cells grown under control culture conditions attached to the culture dish, they frequently formed spherical cell aggregates resembling neurospheres (Figure $4 \mathrm{H})$. This tendency was markedly increased when the cells were grown in serum-free culture media (Figure 4I). Under these conditions, many of the cells in the aggregates became nestin positive (Figure 4J). The cell aggregates were loosely attached to the culture surface and/or floated in the medium, a characteristic of neurospheres and spheres derived from embryonic stem cells. Transfer of a cell aggregate to a new dish with standard media caused the cells to proliferate. Seeding of the cells on fibronectin markedly inhibited formation of cellular spheres by papillary cells.

Characterization of renal papillary cell clones. Three independent clones of renal papillary cells were established using standard single-cell cloning methods and all provided identical results. As shown in Figure 5, A and B (left panels), in standard culture conditions, cells were myofibroblastlike and spindle-shaped and expressed mesenchymal proteins such as $\alpha$-smooth muscle actin. However, the cells also coexpressed epithelial proteins such as ZO-1 (Figure $5 \mathrm{~A}$, right). Furthermore, when the culture conditions were changed, the cell progeny expanded (Figure 5B); during control culture conditions without fetal bovine serum, all cells expressed $\alpha$-smooth muscle actin and were spindleshaped (Figure 5B, left), but in the presence of leukemia inhibitory factor (LIF), the majority of cells became negative for $\alpha$-smooth muscle actin and formed a monolayer, while a small number of cells retained the myofibroblast phenotype, expressing $\alpha$-smooth muscle actin, and grew on top of the monolayer (Figure 5B, right).

Another study further suggested that these cells can give rise to more than one cell type when the cells were examined using neuronal markers. As shown in Figure 5C, individual clones gave rise to heterogeneous cell populations, with some cells expressing nestin, a marker of neuronal stem cells (18), and some cells were found to be positive for class III $\beta$-tubulin, a marker of fully differentiated neurons (19). Indeed, on occasion, some cells even acquired morphological characteristic of neurons. These results suggest that the isolated papillary cells were pluripotent.

Response of papillary BrdU-retaining cells during kidney repair. If the BrdU-retaining papillary cells were adult renal stem cells, they might be involved in kidney repair. To test this, we induced transient unilateral renal ischemia and examined the abundance of BrdU-positive cells in the papillae of both kidneys after kidney recovery (2). Analysis of papillary sections $100 \mu \mathrm{m}$ in thickness (Figure 6A) revealed that at 3 weeks after ischemia there was a marked reduction in the number of BrdU-positive cells in the papilla of the ischemic kidney. Indeed, in 5- $\mu \mathrm{m}$ sections (Figure 6B), the papillary tip of the control kidney showed numerous BrdU-retaining cells, whereas that of the ischemic kidney had very few BrdU-positive cells.

Quantification of this observation was obtained by flow cytometry. Figure $6 \mathrm{C}$ shows the results obtained with 6 rats that underwent transient renal ischemia in the left kidney followed by analysis of cells from both kidneys 3 weeks later. As shown, $36 \%$ of the cells from control papillae were positive for BrdU (i.e., in the M1 region), while only $4 \%$ were positive in papillae from the ischemic kidneys. One possibility that could account for the disappearance of the papillary BrdU-retaining cells after transient renal ischemia is that the cells died. The renal papilla is believed to have a $\mathrm{pO}_{2}$ as low as $4-10 \mathrm{~mm} \mathrm{Hg}(20)$, and its cells might be more vulnerable to ischemia. To rule out this pos- 


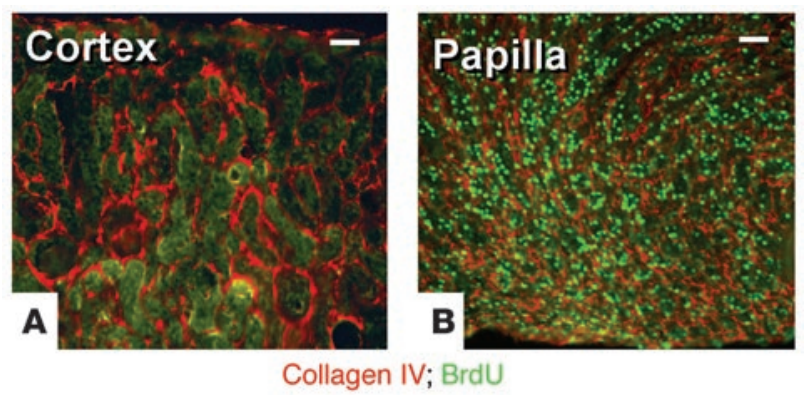

Figure 3

BrdU-retaining cells in adult mouse kidney. (A and B) Composite views $60 \mu \mathrm{m}$ in thickness of kidney sections from a 2-month-old mouse obtained by confocal microscopy with 2- $\mu$ m optical sections. (A) Outer cortex with no BrdU-positive cells. (B) Outer papilla with abundant BrdU-retaining cells. Scale bars: $50 \mu \mathrm{m}$.

sibility, we examined the kidneys of 4 rats for the presence of apoptosis 24 hours after transient ischemia using TUNEL staining of fragmented DNA. Figure 7 shows sections from a representative kidney; apoptosis was prominent in the renal cortex (not shown) and medulla (Figure 7, left). There was an abrupt decrease of the apoptotic signal in the transitional zone from medulla to papilla (Figure 7, middle), and the papilla (Figure 7, right) had essentially no TUNEL staining. This indicates that the renal papilla was more resistant to the ischemic insult than other parts of the kidney, a finding consistent with previous morphological observations (21).

Another possibility for the disappearance of the papillary BrdUpositive cells is that they migrated to the medulla and cortex. However, the number of BrdU-positive cells in the medulla or cortex in the kidneys subjected to ischemia remained the same, ruling out this possibility.

Papillary cell proliferation during renal repair. The absence of significant apoptosis in the papilla of kidneys that underwent transient ischemia, together with the decrease in the number of papillary BrdU-retaining cells, suggested that these cells prolif-

\section{Figure 4}

Renal papillary cells grown in cell culture. (A) Isolated renal papillary cells grown in standard cell culture conditions formed cell aggregates within about 24 hours of their isolation and expressed ZO-1 in their tight junctions. (B and C) Phase-contrast (Phase; B) and fluorescence (C) microphotographs of a group of renal papillary cells growing in cell culture conditions 4 days after cell isolation; about $40 \%$ of the papillary cells isolated from BrdU-loaded animals were BrdU-retaining cells. (D and $\mathbf{E}$ ) During the first several days of culture, most cells had an epithelial phenotype expressing ZO-1 in their tight junctions (D), but some cells, in addition to ZO-1, also expressed mesenchymal proteins such as $\alpha$-smooth muscle actin ( $\alpha \mathrm{SMA})(\mathrm{E})$. (F) After more than a week in culture under control conditions with $5 \% \mathrm{CO}_{2}$ and $95 \%$ room air, most cells were spindle-shaped and stained strongly for $\alpha$-smooth muscle actin. (G) However, when cells were grown under hypoxic conditions $\left(5 \% \mathrm{CO}_{2}\right.$, $1.5 \% \mathrm{O}_{2}$, and $93.5 \% \mathrm{~N}_{2}$ ), most cells retained an epithelial phenotype, with prominent ZO-1 expression. (H) When grown in standard control cell culture media on plastic, most cells adhered to the culture dish but frequently formed cell aggregates that resembled neurospheres. (I) The tendency for the cells to aggregate was markedly enhanced by growth of the cells in the absence of sera. The picture shows a 3-week-old culture with many cellular aggregates. (J) Many of the cells inside the aggregates were positive for nestin. Scale bars: $50 \mu \mathrm{m}$.

erated and that the loss of BrdU signal was due to dilution of the dye in the daughter cells. To test this, we first examined whether cellular proliferation could be detected in the renal papilla after ischemia. Two independent methods were used. In the first, transient unilateral renal ischemia was induced in rats and, 36 hours later, both kidneys were harvested and their papillae were examined for the presence of the marker for proliferating cells, Ki-67 antigen (22). A representative example of four experiments is shown in Figure 8A. While the papilla from the control kidney had essentially no signal, Ki-67 antigen was easily detectable in the papilla of the ischemic kidney, indicating that many papillary cells had entered the cell cycle.

In the second method, cellular proliferation was assayed by DNA synthesis. We induced transient unilateral renal ischemia in rats and, 36 hours later, administered one dose of BrdU to the animals an hour before euthanasia; thus, in this protocol of "acute" BrdU labeling, brightly staining cells were the cells that synthesized DNA during this hour. Figure 8, B and C, shows results from both kidneys of two such experiments. Analysis of $100-\mu \mathrm{m}$ sections showed that there were proliferating cells in the cortex and medulla of both the control kidney and ischemic kidneys, but the latter had many more proliferating cells, particularly in the medulla (not shown). However, in the papilla of the post-ischemic kidney there were abundant dividing cells (labeled during the $1 \mathrm{~h}$ of BrdU administration), while the control kidney papilla had only a few such cells (Figure 8B). Prolif-
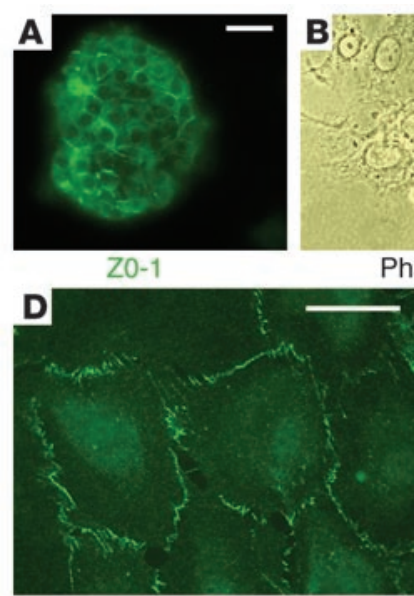

$\mathrm{ZO}-1$

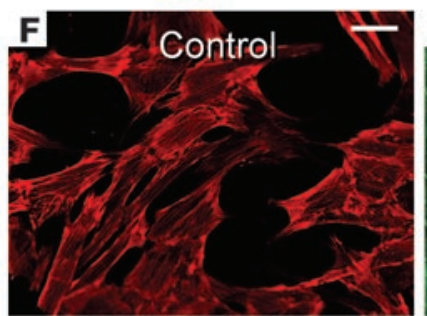

$\alpha \mathrm{SMA}$

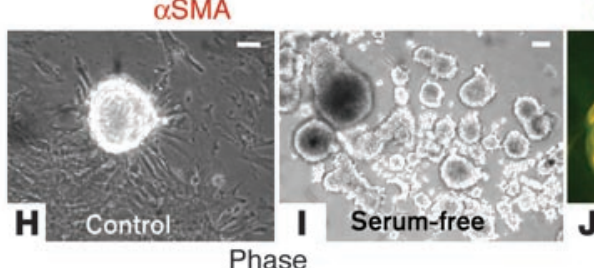

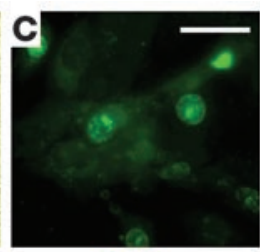

BrdU

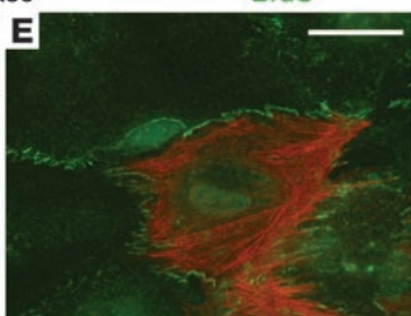

Z0-1; $\alpha$ SMA

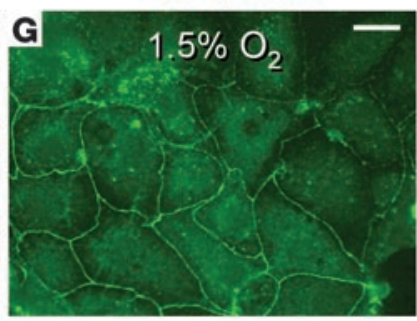

$\mathrm{ZO}-1$ 
A

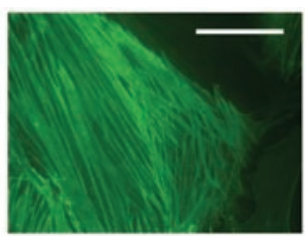

aSMA

B

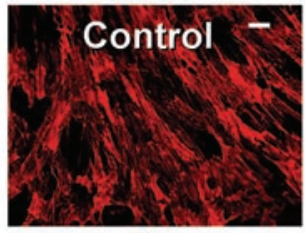

$\alpha \mathrm{SMA}$

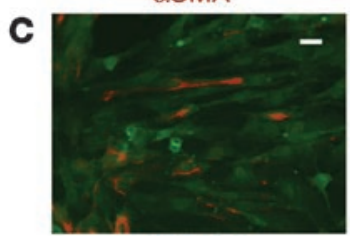

Nestin

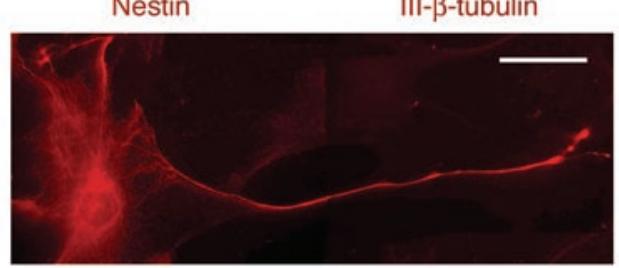

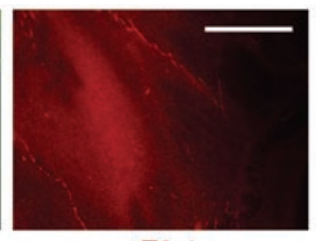

ZO-1
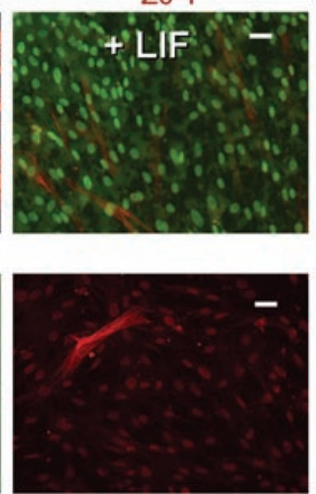

III- $\beta$-tubulin eration was not randomly distributed in the papilla; it was most prominent in the outer papilla, particularly in the region next to the urinary space (i.e., the periphery of the outer papilla; Figure $8 \mathrm{C})$. One possible explanation for this location is that the dividing stem cells (called "transit-amplifying cells" in other organs; ref. 10) were migrating toward the medulla. The topology of the papilla suggests that should it act a source for migration of cells into the medulla, its periphery may be an ideally central location for efficient distribution, as the medulla has a much larger

\section{Figure 6}

Effect of transient renal ischemia on papillary BrdU-retaining cells. The presence of BrdU-retaining cells was examined in papillae of control kidneys and of kidneys subjected to transient ischemia. (A) In 100- $\mu \mathrm{m}$ papillary sections 3 weeks after ischemia, compared with control, there was a marked decrease in BrdU-retaining cells in the papilla of the ischemic kidney. (B) Sections $5 \mu \mathrm{m}$ in thickness showed that while the papillary tip of the control kidney had numerous BrdU-retaining cells, that of the ischemic kidney had very few. (C) For quantification of the effect of renal ischemia on the number of BrdU-retaining cells, 6 rats 5 months of age were subjected to transient ischemia of the left kidney; 3 weeks later their kidneys were harvested and cells from different parts of both kidneys were stained for BrdU and analyzed by flow cytometry. In all graphs, the $y$ axis shows the number of cells, while the $x$ axis (FL2-H) shows the fluorescent intensity. MI is the area of positive cells. In the papillae of the nonischemic kidneys, the number of BrdU-retaining cells averaged $36 \%$ of the total cells, while in the papillae of the kidneys subjected to transient ischemia, this number was only $4 \%$, as shown to the left of each histogram. Compared with the control kidney, the ischemic kidney also had a slight decrease in the number of BrdU-positive cells in the medulla and cortex, suggesting that the papillary BrdU-retaining cells had not simply migrated to other parts of the kidney. Scale bars: $50 \mu \mathrm{m}$.

\section{Figure 5}

Characterization of renal papillary cell clones. (A) As the example shows, many cells coexpressed mesenchymal proteins such as $\alpha$-smooth muscle actin (left) as well as epithelial proteins such as ZO-1 (right). (B) In standard control conditions in the absence of sera, all cells were spindle-shaped and stained strongly for $\alpha$-smooth muscle actin (Control; left) but when LIF was added to the media, two phenotypes were apparent (+ LIF; right): the majority of cells grew in a monolayer and were negative for $\alpha$-smooth muscle actin (nuclei stained with $10 \mathrm{nM}$ Sytox green), but a few cells grew on top of the monolayer of cells, were spindle-shaped, and expressed $\alpha$-smooth muscle actin. (C) In standard control culture conditions, a small number of cells expressed the intermediate filament nestin. Occasional cells also expressed the neuronal marker class III $\beta$-tubulin (III- $\beta$-tubulin) and some cells even acquired the morphological characteristics of neurons. Scale bars: A, $20 \mu \mathrm{m}$; B and C, $50 \mu \mathrm{m}$.

volume and the distance from the outer papilla to any point in the medulla is the shortest possible.

Papillary BrdU-retaining cells proliferate during renal repair. To directly examine whether the proliferation of the papillary cells that occurs after transient ischemia includes the BrdU-retaining cells, we used rats that had been "loaded" with BrdU during the postnatal period and harvested their kidneys 36 hours after transient ischemia. Sections of the renal papilla were examined for both Ki-67 antigen and BrdU. Figure 9 depicts an example of four such experiments. Figure 9A shows one section with BrdU staining, Ki-67 staining, and the merged image, indicat-

A

$100 \mu \mathrm{m}$

B

C

Papilla

$36 \%$

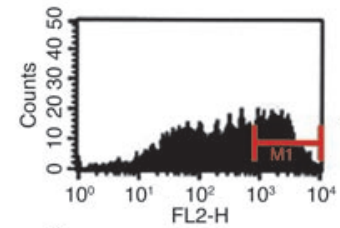

Medulla

$7 \%$
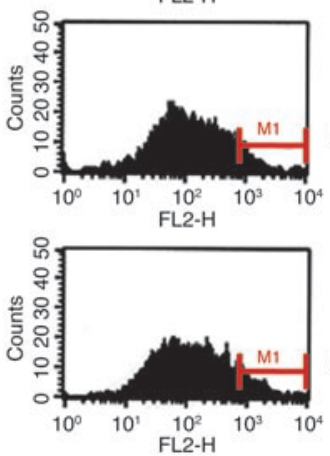

Cortex

$8 \%$
Ischemic
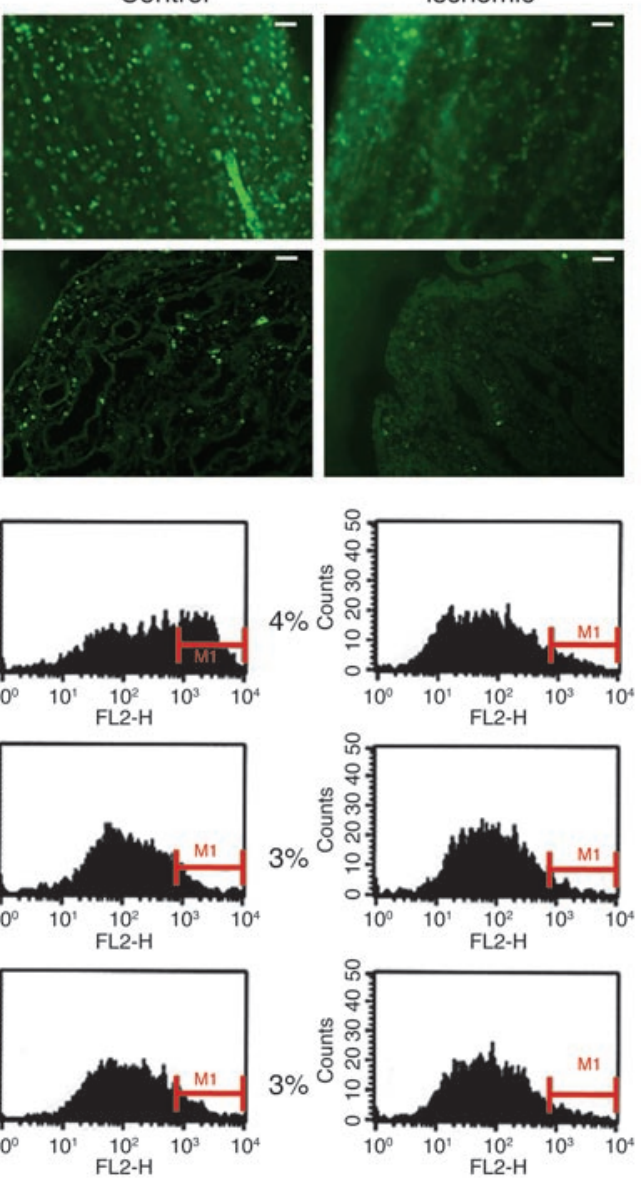

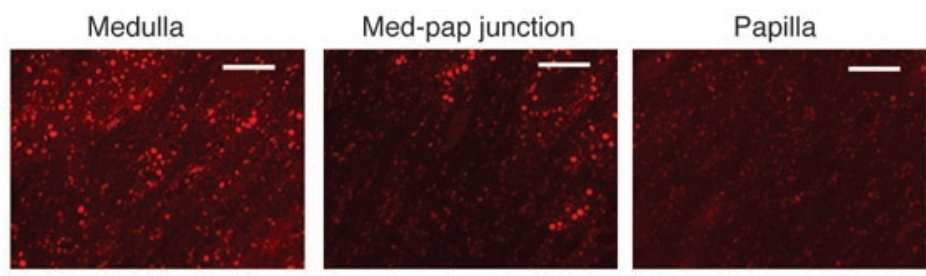

Figure 7

TUNEL staining of apoptotic cells after transient renal ischemia. Representative example of 5-um sections of different areas of the same kidney 24 hours after ischemia. All three photographs were obtained under identical conditions. The papilla is the only part of the kidney with no significant apoptotic signal. Med-pap, medullary-papillary. Scale bars: $50 \mu \mathrm{m}$.

ing that many BrdU-retaining cells also expressed the proliferation marker Ki-67. This is more clearly shown in the two higherpower microphotographs of Figure 9B. Note that some papillary cells were positive only for BrdU, others contained both signals (i.e., were BrdU-retaining cells that were proliferating), while still others displayed only the Ki-67 signal. These latter cells could be cells that never retained BrdU or could be progeny of BrdUretaining cells that had lost the signal as the nucleotide became diluted during cell division.

Intrarenal injection of papillary cells. If renal papillary cells participate in renal repair, these cells or their progeny should be capable of migrating toward and incorporating into other parts of the kidney. To test this, we labeled renal papillary cells grown in vitro with the fluorescent dye PKH26 and injected them into the subcapsular area of the left kidney (23). Seven days later, both kidneys were harvested and sections were analyzed for the presence of labeled cells. In five such experiments, labeled cells were detected only in the injected kidney, in which groups of cells frequently concentrated into a self-limited nodule in the subcapsular region or the renal cortex (not shown). However, the cells also spread to other parts of the kidney such as the medulla, as shown in the representative experiment depicted in Figure 10. Note that some labeled cells (rhodamine) were present in the renal interstitium (Figure 10B, arrows), but other cells resided inside the tubules (Figure 10, B-D), identified by staining of the collagen IV in their basement membrane (FITC fluorescin).

\section{Figure 8}

Cellular proliferation in the renal papilla after transient renal ischemia. (A-C) For detection of whether cellular proliferation occurs in the renal papilla after transient renal ischemia, 36 hours after the episode, kidneys were harvested and papillae were examined. (A) Control and ischemic papillae of the same animal stained with an antibody against the proliferation cell marker Ki-67 (rhodamine). Only the papillae of the post-ischemic kidney showed significant proliferation. (B and $\mathbf{C}$ ) Control and ischemic papillae of two different experiments in which rats were given a dose of BrdU 1 hour before euthanasia and sections of papilla were examined for BrdU incorporation as marker of DNA synthesis. Sections of renal papillae are $100 \mu \mathrm{m}$ in thickness. (B) Papillary sections from the outer or juxtamedullary area (top), middle area (middle), and tip (bottom) from control and ischemic kidneys. As shown, DNA synthesis was only detected in the papilla of the postischemic kidney, particularly in the outer papilla, the area next to the medulla. (C) In the kidney subjected to transient ischemia, DNA synthesis in papillary cells was most prominent in the region adjacent to the urinary space of the outer papilla. Scale bars: $50 \mu \mathrm{m}$.

\section{Discussion}

Our results indicate that the renal adult papilla contains large numbers of slowly cycling cells (i.e., BrdU-retaining cells) that persist throughout the life of the animal but quickly enter the cell cycle and disappear from the papilla during recovery from a transient episode of ischemia, suggesting that these cells participate in renal repair. The disappearance of the cells from the papilla could not be explained by their death in response to ischemia; apoptosis was absent from the papilla but was abundant in other renal regions after ischemia. This suggests that, as in other organs (10), proliferating cells may migrate toward the site of maximal injury (i.e., the medulla). In addition, renal papillary cells grown in vitro were capable of incorporating into other parts of the renal parenchyma, including renal tubules. Isolation and in vitro culture of papillary cells allowed derivation of clones capable of generating more than one cell type, which, like metanephric mesenchymal stem cells (13), showed remarkable plasticity and coexpressed epithelial and mesenchymal markers. Furthermore, the cells were capable of forming spheres, a characteristic of many organ-specific adult stem cells in vitro (15-17). As an aggregate, these data indicate that adult kidney stem cells reside in the papilla.

Although we demonstrated that isolated renal papillary cells were pluripotent and the BrdU-retaining cells were located in the papilla, the fraction of these renal papillary cells that may be stem cells is unknown. Several technical issues limited our ability to equate stem cells with BrdU-retaining cells, including problems

A

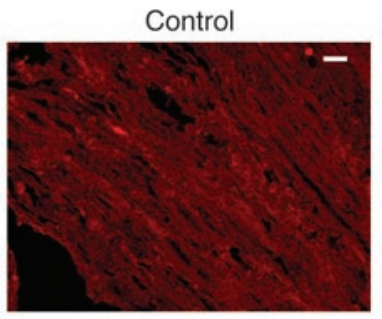

B

Outer

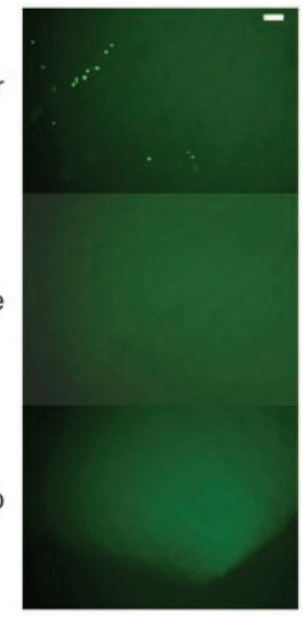

C

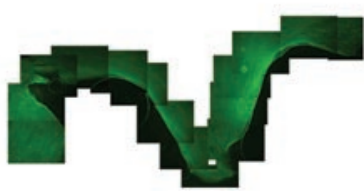

Ischemic
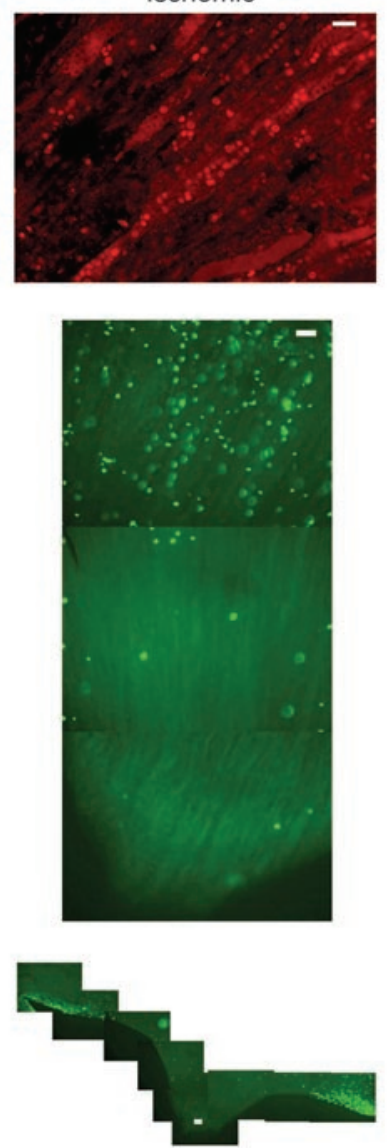


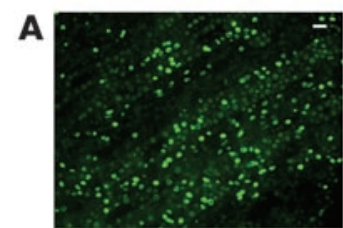

BrdU

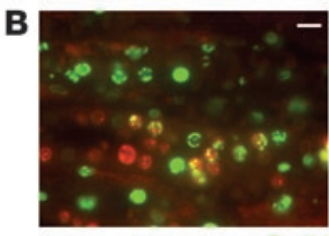

BrdU; Ki-67

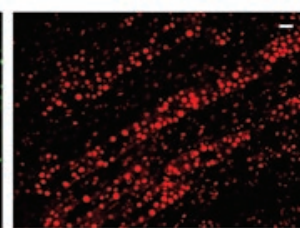

$\mathrm{Ki}-67$

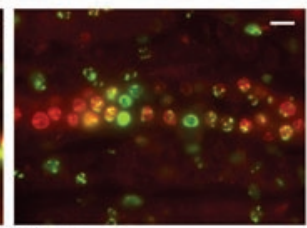

Figure 9

Cellular proliferation of papillary BrdU-retaining cells after transient renal ischemia. (A and B) For determination of whether the papillary cell proliferation observed after ischemia involved the BrdU-retaining cells, transient ischemia was induced in adult rats loaded with BrdU when they were pups. Then, 36 hours after the ischemia, the kidneys were harvested and $5-\mu \mathrm{m}$ sections of their papillae were examined for BrdU (FITC fluorescin) and the Ki-67 antigen (rhodamine). In the far right panel of $\mathbf{A}$, and the higher power views of $\mathbf{B}$, merged images show that many BrdU-retaining cells began cycling after ischemia. Scale bars: $50 \mu \mathrm{m}$.

regarding incorporation of BrdU or its detection in the cells that retain it. First, it is not currently possible to demonstrate that a pluripotent cell in vitro is also a low-cycling one in vivo, as BrdU detection is a destructive procedure. Second, the BrdU labeling method is unlikely to detect all stem cells, as BrdU incorporates only during the $\mathrm{S}$ phase of the cell cycle and only if all the cells were synchronous would we expect them to be equally labeled. Furthermore, we do not know the length of the cycle in these cells, and studies in other stem cells have shown that their cell cycle lengthens as the organ matures (24). Thus, there are too many unknown factors to predict with any degree of accuracy the fraction of stem cells that would be labeled by a short pulse of BrdU and how many of the papillary cells are stem cells. Indeed, quantification of the stem cells in the renal papilla will require their isolation and identification using methods that do not damage them (e.g., specific cell surface markers).

The vast body of knowledge of the hematopoietic stem cell has dominated the intellectual landscape of adult organ-specific stem cells. Although in the bone marrow a single cell generates all the cellular components of circulating blood, it appears unlikely that an adult renal stem cell could generate a complete nephron de novo. In solid organs with complex structures such as the kidney, it appears more likely that adult stem cells have a more restricted generative capacity and that their function may be limited to replacement of isolated dying cells during organ maintenance or after injury repair. If this is the case, it will be

\section{Figure 10}

Intrarenal injection of renal papillary cells. (A-D) Papillary cells were labeled with PKH26 (rhodamine) and were injected into the subcapsular space. Seven days later, sections $3 \mu \mathrm{m}$ in thickness showed that many cells had scattered into the renal medulla $(\mathbf{A})$. Some cells were located in the interstitial space (arrows in B), but many incorporated inside tubules (arrowheads, B-D), which are highlighted by staining of the collagen IV (FITC fluorescin) in their basement membranes. Scale bars: $50 \mu \mathrm{m}$. of great interest to determine whether a single stem cell may replace cells in different nephron segments or whether different stem cells are required for each terminally differentiated cell type.

It is striking that the BrdU-retaining papillary cells were both interstitial cells and well differentiated epithelia (Figure 3A). However, in other organs, adult stem cells are not necessarily amorphous and undifferentiated. Indeed, adult neuronal stem cells display morphological and molecular characteristics of differentiated astrocytes (25). In addition, de-differentiation of renal epithelial cells into myofibroblasts, a long-suspected process (26), has recently been demonstrated conclusively (27), and it may be postulated that, as during limb and tail repair in some amphibians (28), de-differentiation of renal epithelial cells after organ injury may be followed by cell proliferation and re-differentiation into multiple cell types (29). Thus, terminally differentiated kidney cells may well be functioning as pluripotent stem cells during organ maintenance and repair.

Needless to say, a critical question is whether the rare BrdU-retaining cells that were scattered throughout the cortex and medulla originated in the papilla or were an entirely different cell population. Organ-specific stem cells have substantial migratory capacity $(10,30)$, but it is currently unknown whether upon migration outside their niche they retain their characteristic phenotype (e.g., slow cycling time). New methods of isolation of renal BrdU-retaining cells will be needed in order to address these questions. Regardless, the number of BrdU-retaining cells in the cortex and medulla decreased slightly after recovery from transient ischemia, suggesting that whatever their origin, these cells also participated in renal repair.

While the adult kidney contains small numbers of BrdU-retaining cells throughout its parenchyma, it is clear that the papilla is a reservoir for such cells and that this region of the kidney is where the renal stem cell niche is probably located. A stem cell niche is a restricted environment where presumably the factors that are needed to control growth and differentiation abound, allowing

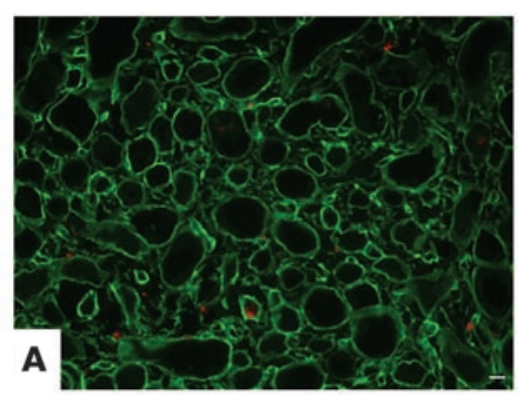

PKH26; collagen IV
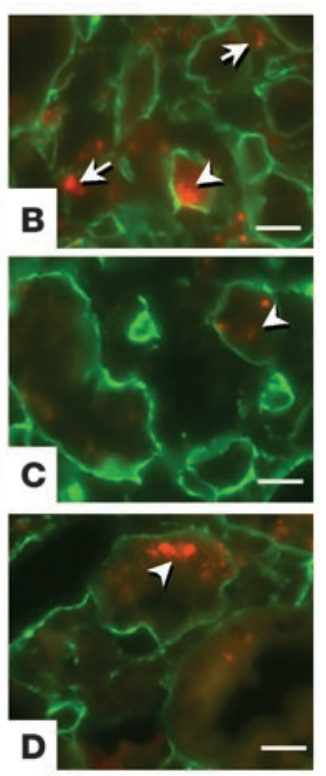
stem cell protection, self-renewing capacity, and differentiation. Our finding that after ischemia the renal papilla is the only part of the kidney where apoptosis could not be detected is consistent with the notion that it provides a protective niche for stem cells. Similarly, in their niche, neuronal stem cells concentrate around blood vessels (11), and the papillary BrdU-retaining cells were in close association with endothelial cells (Figure 2I). Studies in the Drosophila ovary have elucidated many of the molecular signals responsible for these functions, but understanding of mammalian stem cell niches is only beginning $(31,32)$. Thus, the finding that the renal papilla, with its unique environment, is a likely renal stem cell niche is of great interest. The renal papilla has the highest osmolality of any part of the body, and its $\mathrm{pO}_{2}$ is believed to be as low as $4-10 \mathrm{~mm} \mathrm{Hg}$ (reviewed in ref. 20). While a potential role for the hypertonicity of the papilla in the control of the precursor cells remains to be explored, a role for hypoxic microenvironments in the control of adult organ-specific stem cell function has long been suspected (33), and recent work with bone marrow (34) and neuronal stem cells (35) has shown that low oxygen tension enhances their survival, proliferation, and capacity to differentiate. Moreover, the transcriptional regulator hypoxia-inducible factor- $1 \alpha$ (HIF- $1 \alpha)$, which activates many of the cellular responses to low oxygen tension, has a central role in these responses $(34,36)$. Expression of HIF-1 $\alpha$ in the kidney during hypoxia is most pronounced in the papilla, where its distribution strikingly resembles the distribution of the BrdU-retaining cells (37). In addition, after transient renal ischemia there is little or only a modest increase in the expression of HIF- $1 \alpha$ in the renal cortex and medulla, but in the renal papilla the expression of this transcription factor increases markedly (34). These data, together with our findings that hypoxia modulates the phenotype of isolated papillary cells (Figure 6, C and D) and that the renal papilla, unlike other parts of the kidney, displayed no apoptosis after transient ischemia (Figure 7), strongly suggest that oxygen tension and hypoxia-activated genes have a critical regulatory function in renal papillary stem cells.

Traditionally, organ-specific adult stem cells are defined as resident cells that can self-renew and generate more than one terminally differentiated cell type. While the BrdU-retaining cells in the papilla are very likely the stem cells of the adult kidney, confirmation that these cells conform in vivo to the traditional definition of stem cells will require additional work. However, the molecular parameters that define a stem cell are unknown at present and, given that the concepts of organ-specific stem cell and organ maintenance and repair are intrinsically linked, what is needed is a detailed understanding of the origin of new cells in the adult kidney (38) more than demonstration that renal precursor cells conform to the classical definition of "stemness."

Despite many uncertainties, it is already apparent that such understanding will reveal that the generation of new adult kidney cells occurs by multiple mechanisms. Morphological analysis of kidney repair after injury has long suggested that some cells generate by division of terminally differentiated cells $(3,4)$. In this context, a recent publication by Maeshima et al. (39) is of interest. Those workers gave BrdU to adult rats for a week and sacrificed the animals 2 weeks later. In normal kidneys, they found scattered labeled cells in the proximal, distal, and collecting tubules, cells that must have divided during the week of BrdU administration and during the subsequent 2 weeks either had not divided or had divided rarely. This finding is entirely consistent with previous studies showing that mitotic figures in randomly selected kidney sections exist in about 1:1,000 cells and indicate a modest rate of cell division in the adult kidney. More interesting is their additional observation that some of the BrdU-labeled cells in tubules divided after ischemia, adding additional support to the contention that after injury to the kidney, renal cells can be generated from division of terminally differentiated cells.

Another potential origin for new, terminally differentiated kidney cells during kidney repair may be the mesenchymal cells that develop during the epithelial to mesenchymal transition, which occurs during renal injury (27), as found in other systems (28). A surprising recent finding has been the discovery that some of the new renal cells are derived from extrarenal cells. Several groups have shown that bone marrow cells can colonize the kidney and contribute to its repair (5-7). It remains to be established whether these invading cells are already true stem cells capable of generating multiple renal cell progeny or are terminally differentiated cells that upon entering the kidney's stem cell niche are genetically reprogrammed to assume the kidney cell differentiation pathway. Finally, although it is clear that the papillary BrdU-retaining cells proliferate during kidney repair and are very likely "stem cells," the cell progeny of these cells in vivo needs to be established.

While interest in the renal papilla has long centered on its critical role in osmotic regulation, our findings shed a new light on this part of the kidney. We believe that the response of the papilla to renal injury may well hold clues to several previously mysterious events in the kidney's response to a number of diseases. For example, many patients with sickle cell anemia develop renal disease and about 5\% will need dialysis over the course of their lifetime (40-42). Although papillary abnormalities including necrosis have long been recognized as a frequent complication of this disease, the renal disease they develop includes proteinuria and glomerulosclerosis, both diseases of the renal cortex. Similarly, it has been suggested based on clinical studies that global renal damage results from other forms of papillary necrosis $(43,44)$. Perhaps it is the loss of papillary renal stem cells that leads to the global renal damage in these diseases because of the lack of enough cells to repopulate damaged nephrons. Furthermore, the capacity of the kidney to recover its function after prolonged ischemia (2) could be due to the papilla's resistance to the ischemic damage (Figure 7 and ref. 21). Similarly, the administration of nephrotoxic compounds such as mercuric chloride, which induces transient acute renal failure, is associated with marked morphologic changes in the renal papilla, but these changes suggest increased cellular activity rather than cellular injury (45).

\section{Methods}

BrdU loading of rats and mice. To obtain animals with BrdU-retaining cells, 3-day-old Sprague-Dawley rats and CD 1 mice were given $50 \mu \mathrm{g} / \mathrm{g} \mathrm{BrdU}$ (Sigma-Aldrich) subcutaneously twice daily for 3.5 days. Afterward, animals were allowed to grow and their kidneys were harvested starting at 2 months of age. Euthanasia was accomplished by $\mathrm{CO}_{2}$ narcosis.

Transient renal ischemia. Sprague-Dawley rats weighing about $300 \mathrm{~g}$ were anaesthetized with ketamine/xylazine and a posterior subcostal incision was made in the left side and the renal artery was dissected. A small vascular clamp was used to occlude the renal artery for 45 minutes while the animal was kept at $37^{\circ} \mathrm{C}$ with a heating blanket. After removal of the clamp, the wound was sutured and the rat was returned to the cage. In the rats in which cellular proliferation was examined after ischemia with BrdU, rats were given a single dose of $100 \mathrm{mg} / \mathrm{kg}$ of BrdU intraperitoneally 1 hour prior to being sacrificed. All animal studies have been approved by The Institutional Animal Care and Use Committee at Columbia University. 
Isolation of renal papillary cells. For isolation of papillary cells, kidneys were harvested and kept in HBSS containing $15 \mathrm{mM}$ HEPES, penicillin/ streptomycin, and $0.35 \mathrm{~g} / 1 \mathrm{NaHCO}_{3}, \mathrm{pH} 7.4$, at $4^{\circ} \mathrm{C}$. After removal of the perinephric fat, the kidneys were sectioned along the anterior-posterior axis, ensuring that the papilla was left intact and attached to one of the two half-kidneys. After isolation of the papilla, the tissue was minced and digested with $2 \mathrm{mg} / \mathrm{ml}$ collagenase I (Worthington) while being shaken at $175 \mathrm{rpm}$ in a $37^{\circ} \mathrm{C}$ shaker. Thereafter, with a spatula, the tissue was forced through sequential 106- $\mu \mathrm{m}$ and $20-\mu \mathrm{m}$ steel filters (VWR). The dispersed cells were then collected by centrifugation.

Cell culture. For tissue culture, the collected cells were dispersed with DMEM/Ham F12 (Invitrogen Corp.) containing 10\% FCS, 5\% chicken embryo extract, and 5\% rat serum (both from USBiological), as well as $2 \mathrm{mM}$ glutamine, penicillin, and streptomycin. Except where indicated otherwise, all cultures were maintained at $37^{\circ} \mathrm{C}$ in an atmosphere of $5 \%$ $\mathrm{CO}_{2}$ and $95 \%$ room air. When cells were grown in serum-free media, the culture media consisted of DMEM/Ham F12 containing glutamine and antibiotics plus $5 \mu \mathrm{g} / \mathrm{ml}$ insulin, $5 \mu \mathrm{g} / \mathrm{ml}$ transferrin, $5 \mathrm{ng} / \mathrm{ml}$ sodium selenite, $20 \mathrm{ng} / \mathrm{ml}$ dexamethasone, $20 \mathrm{ng} / \mathrm{ml}$ l-thyroxine (all from SigmaAldrich), $50 \mathrm{ng} / \mathrm{ml} \mathrm{bFGF,} 100 \mathrm{ng} / \mathrm{ml}$ PDGF, and $20 \mathrm{ng} / \mathrm{ml} \mathrm{EGF}$ (all from R\&D Systems). Cells were grown either in plastic dishes or in dishes coated with $5 \mu \mathrm{g} / \mathrm{cm}^{2}$ fibronectin (BD). When used, LIF (R\&D Systems) was added at a concentration of $100 \mathrm{ng} / \mathrm{ml}$. Individual cell clones were obtained as described (13).

Flow cytometry. For flow cytometry of renal papillary cells, kidney papillae were digested as described above and the collected cells were suspended in PBS containing $10 \mathrm{U} / \mathrm{ml}$ DNAse I (Roche), 3\% FCS, and 1\% donkey serum. At $4{ }^{\circ} \mathrm{C}$, cells were incubated with primary antibodies and, where needed, after centrifugation and washing, with secondary antibodies coupled with fluorophorea. Prior to flow cytometry, cells were washed and incubated with propidium iodide (Molecular Probes).

For flow cytometry of cells from different regions of the kidney, in addition to the papilla, the cortex and medulla were dissected. Tissue samples from all three regions were digested and their cells were isolated as described above for papillary cells. The collected cells were suspended in PBS containing $10 \mathrm{U} / \mathrm{ml}$ DNAse (Roche). Cells were next fixed at $4^{\circ} \mathrm{C}$ for $30 \mathrm{~min}$ with $70 \%$ ethanol and were processed for $\mathrm{BrdU}$ detection with anti-BrdU-FITC fluorescin (Roche) as detailed by the manufacturer. Data were collected with a FACStarPlus cytometer (BD). A minimum of 10,000 cells were collected for all analyses. Gating was constructed based on negative controls, and compensation controls were included in all analyses performed. Population percentages and numbers were generated for gated populations from each experiment using CellQuest software (BD).

Immunodetection. Kidneys were isolated and sectioned on the anterior-posterior axis, with care taken to ensure that the papilla remained in one of the two half-kidneys. For $5-\mu \mathrm{m}$ sections, the half-kidney containing the papilla was fixed with either $4 \%$ paraformaldehyde or $10 \%$ formalin. In the former case, after fixation the kidney was incubated with $30 \%$ sucrose in PBS and afterward was frozen in Tissue-Tek OCT compound (Sakura Finetek) with a slurry of solid $\mathrm{CO}_{2}$ in 2-methylbutane. When the kidneys were fixed in $10 \%$ formalin, they were processed into paraffin blocks by standard techniques. For $100-\mu \mathrm{m}$ sections, the half-kidney containing the papilla was first fixed in $-20^{\circ} \mathrm{C}$ methanol and afterward was embedded in a block of $3 \%$ agarose. Sections were obtained with a 1000 Plus Vibratome (Pelco).

BrdU was detected with a FITC fluorescin-coupled mouse monoclonal antibody against BrdU (Roche) following the manufacturer's procedure, except where indicated otherwise (see below). Briefly, sections were incubated with $2 \mathrm{~N} \mathrm{HCl}$ at $37^{\circ} \mathrm{C}$ for 1 hour, then buffered with $0.1 \mathrm{M}$ sodium borate, $\mathrm{pH} 8.5$, followed by incubation in PBS. All unlabeled primary antibodies were detected as described previously (13).
Cell proliferation in rats "loaded" with BrdU at 3 days of age was assayed in paraffin sections by immunodetection with a monoclonal antibody against rat Ki-67 antigen (22). Briefly, after being dewaxed, sections were incubated for 45 minutes at $95^{\circ} \mathrm{C}$ with DakoCytomation Target Retrieval Solution (DAKO). After being rinsed with PBS, sections were incubated with the antibody against Ki-67 and afterward with a secondary antibody against mouse, coupled to rhodamine. Next, sections were incubated for 1 hour at room temperature with $10 \mathrm{U} / \mathrm{ml}$ of DNAase I (Roche) in $25 \mathrm{mM}$ Tris and 50\% glycerol, pH 7.6. After being rinsed, sections were finally incubated with the FITC fluorescin-coupled antibody against BrdU described above. Detection of apoptosis was performed in paraffin sections by TUNEL staining of fragmented DNA with the In Situ Cell Death Detection Kit TMR red (Roche) following the instructions of the manufacturer.

Fluorescent signals were detected with a fluorescence microscope and an RT Slider SPOT digital camera (Diagnostic Instruments Inc.). Sections were also viewed with an Axiovert 100 laser-scanning confocal microscope (model LSM 410; Carl Zeiss Inc.). Excitation was accomplished with an argon-krypton laser producing lines at $488 \mathrm{~nm}$ for the FITC fluorescinlabeled sample and $568 \mathrm{~nm}$ for the rhodamine-labeled sample. The images were collected, as indicated in the respective figure legends, from optical sections 1 or $2 \mu \mathrm{m}$ in thickness and were analyzed with Zeiss LSM-PC software. Final images were processed with Adobe Photoshop software.

Intrarenal injection of renal papillary cells. Renal papillary cells grown in vitro were trypsinized and labeled with the PKH26 Red Fluorescent Cell Linker Kit (Sigma-Aldrich) following the manufacturer's instructions. The cells were then seeded at subconfluent density and, after overnight culture, a sample of the cells was examined under the fluorescence microscope to ensure dye incorporation. Prior to injection, cells were recovered by being rinsed with HBSS with $0.02 \%$ EDTA. A total of $0.9 \times 10^{6}$ cells were collected in $60 \mu \mathrm{l}$ of culture media. The left kidney of each anesthetized rat was exposed as described above for transient renal ischemia and, with a dissecting microscope, three injections of $20 \mu \mathrm{l}$ of the media containing the cells were made into the subcapsular space (23) of the outer sagittal edge of both kidney poles and the midlevel of the kidney. After the wound was sutured, the animal was returned to its cage and was euthanized 7 days later.

Antibodies. The following antibodies were used: monoclonal against $\mathrm{BrdU}$ (Roche); monoclonal against $\alpha$-smooth muscle actin, clone 1A4 (SigmaAldrich); monoclonal against neuronal class III $\beta$-tubulin, clone TUJ1 (Babco); monoclonal anti-rat nestin, clone Rat 401 (BD Biosciences - Pharmingen); monoclonal against Ki-67 antigen, clone MIB-5 (DAKO); monoclonal against CD34, clone ICO115 (Santa Cruz Biotechnology); FITC fluorescin-labeled monoclonal against CD44H, clone OX-49 (BD Biosciences - Pharmingen); phycoerythrin-labeled monoclonal anti-rat CD45.2, clone HIS41 (eBioscience); polyclonal against c-Kit (Santa Cruz Biotechnology); polyclonal against ZO-1 (Zymed Laboratories); polyclonal against collagen IV (Biodesign International); and polyclonal against von Willebrand factor (DAKO).

\section{Acknowledgments}

This work was supported in part by National Institute of Diabetes and Digestive and Kidney Diseases grant DK-55388.

Received for publication December 23, 2003, and accepted in revised form July 21, 2004.

Address correspondence to: Juan A. Oliver, Department of Medicine, Columbia University, 630 West 168th Street, New York, New York 10032, USA. Phone: (212) 305-6938; Fax: (212) 3053475; E-mail: jao7@columbia.edu.

O. Maarouf and F.H. Cheema contributed equally to this work. 
1. Witzgall, R., Brown, D., Schwarz, C., and Bonventre, J.V. 1994. Localization of proliferating cell nuclear antigen, vimentin, c-Fos, and clusterin in the postischemic kidney. Evidence for a heterogenous genetic response among nephron segments, and a large pool of mitotically active and dedifferentiated cells. J. Clin. Invest. 93:2175-2188.

2. Finn, W.F., and Chevalier, R.L. 1979. Recovery from postischemic acute renal failure in the rat. Kidney Int. 16:113-123.

3. Oliver, J., MacDowell, M., and Tracy, A. 1951. The pathogenesis of acute renal failure associated with trauma and toxic injury, renal ischemia, nephrotoxic damage and the ischemuric episode. J. Clin Invest. 30:1307-1440.

4. Houghton, D.C., Hartnett, M., Campbell-Boswell, M., Porter, G., and Bennett, W. 1976. A light and electron microscopic analysis of gentamicin nephrotoxicity in rats. Am. J. Pathol. 82:589-612.

5. Gupta, S., Verfaillie, C., Chmielewski, D., Kim, Y., and Rosenberg, M.E. 2002. A role for extrarenal cells in the regeneration following acute renal failure. Kidney Int. 62:1285-1290.

6. Lin, F., et al. 2003. Hematopoietic stem cells contribute to the regeneration of renal tubules after renal ischemia-reperfusion injury in mice. J. Am. Soc. Nephrol. 14:1188-1199.

7. Kale, S., et al. 2003. Bone marrow stem cells contribute to repair of the ischemically injured renal tubule. J. Clin. Invest. 112:42-49. doi:10.1172/ JCI200317856.

8. Cotsarelis, G., Cheng, S.Z., Dong, G., Sun, T.T., and Lavker, R.M. 1989. Existence of slow-cycling limbal epithelial basal cells that can be preferentially stimulated to proliferate: implications on epithelial stem cells. Cell. 57:201-209.

9. Johansson, C.B., et al. 1999. Identification of neural stem cell in the adult mammalian central nervous system. Cell. 96:25-34.

10. Lavker, R.M., and Sun, T.T. 2000. Epidermal stem cells: properties, markers, and location. Proc. Natl. Acad. Sci.U. S. A. 97:13473-13475.

11. Palmer, T.D., Willhoite, A.R., and Gage, F.H. 2000 Vascular niche for adult hippocampal neurogenesis. J. Comp. Neurol. 425:479-494.

12. Polesskaya, A., Seale, P., and Rudnicki, M.A. 2003. Wnt signaling induces myogenic specification of resident $\mathrm{CD}^{4} 5^{+}$adult stem cells during muscle regeneration. Cell. 113:841-852.

13. Oliver, J.A., Barasch, J., Yang, J., Herzlinger, D., and Al-Awqati, Q. 2002. Metanephric mesenchyme contains embryonic renal stem cells. Am. J. Physiol. Renal Physiol. 283:F799-F809.

14. Smith, A.G. 1992. Mouse embryo stem cells: their identification, propagation and manipulation. Semin. Cell Biol. 3:385-399.

15. Bodine, D.M., Crosier, P.S., and Clark, S.C. 1991. Effects of hematopoietic growth factors on the survival of primitive stem cells in liquid suspension culture. Blood. 78:914-920.

16. Reynolds, B.A. and Weiss, S. 1996. Clonal and population analyses demonstrate that an EGFresponsive mammalian embryonic CNS precursor is a stem cell. Dev. Biol. 175:1-13.

17. Li, H., Liu, H., and Heller, S. 2003. Pluripotent stem cells from the adult mouse inner ear. Nat. Med. 9:1293-1299.

18. Lendahl, U., Zimmerman, L.B., and McKay, R.D. 1990. CNS stem cells express a new class of intermediate filament protein. Cell. 60:585-595.

19. Kameda, Y., Kameya, T., and Frankfurter, A. 1993. Immunohistochemical localization of a neuron-specific beta-tubulin isotype in the developing chicken ultimobranchial glands. Brain Res. 628:121-127.

20. Zhang, W., and Edwards, A. 2002. Oxygen transport across vasa recta in the renal medulla. Am.J. Physiol. Heart Circ. Physiol. 283:H1042-H1055.

21. Torhorst, J., de Rougemont, D., Brunner, F.P., and Thiel, G. 1982. Morphology of the renal medulla in ischemic acute renal failure in the rat. Nephron. 31:296-300.

22. Scholzen, T., and Gerdes, J. 2000. The Ki-67 protein: from the known and the unknown. J. Cell. Physiol. 182:311-322.

23. Naito, T., et al. 1996. Macrophage growth factors introduced into the kidney initiate renal injury. Mol. Med. 2:297-312.

24. Lewandowski, T.A., Ponce, R.A., Charleston, J.S., Hong, S., and Faustman, E.M. 2003. Changes in cell cycle parameters and cell number in the rat midbrain during organogenesis. Dev. Brain Res. 141:117-128

25. Alvarez-Buylla, A., Seri, B., and Doetsch, F. 2002. Identification of neural stem cells in the adult vertebrate brain. Brain Res. Bull. 57:751-758.

26. Ng, Y.Y., et al. 1998. Tubular epithelial-myofibroblast transdifferentiation in progressive tubulointerstitial fibrosis in $5 / 6$ nephrectomized rats. Kidney Int. 54:864-876.

27. Iwano, M., et al. 2002. Evidence that fibroblasts derive from epithelium during tissue fibrosis. J. Clin. Invest. 110:341-350. doi:10.1172/JCI200215518.

28. Tsonis, P.A. 2000. Regeneration in vertebrates. Dev. Biol. 221:273-284.

29. Bonventre, J.V. 2003. Dedifferentiation and proliferation of surviving epithelial cells in acute renal failure. J. Am. Soc. Nephrol. 14:S55-S61.

30. Nait-Oumesmar, B., et al. 1999. Progenitor cells of the adult mouse subventricular zone proliferate, migrate and differentiate into oligodendrocytes after demyelination. Eur. J. Neurosci. 11:4357-4366.

31. Zhang, J., et al. 2003. Identification of the haematopoietic stem cell niche and control of the niche size. Nature. 425:836-841.

32. Calvi, L.M., et al. 2003. Osteoblastic cells regulate the haematopoietic stem cell niche. Nature. 425:841-846.

33. Cipolleschi, M.G., Dello Sbarba, P., and Olivotto, M. 1993. The role of hypoxia in the maintenance of hematopoietic stem cells. Blood. 82:2031-2037.

34. Danet, G.H., Pan, Y., Luongo, J.L., Bonnet, D.A., and Simon, M.C. 2003. Expansion of human SCIDrepopulating cells under hypoxic conditions. J. Clin. Invest. 112:126-135. doi:10.1172/JCI200317669.

35. Morrison, S.J., et al. 2000. Culture in reduced levels of oxygen promotes clonogenic sympathoadrenal differentiation by isolated neural crest stem cells. J. Neurosci. 20:7370-7376.

36. Adelman, D.M., Gertsenstein, M., Nagy, A., Simon, M.C., and Maltepe, E. 2000. Placental cell fates are regulated in vivo by HIF-mediated hypoxia responses. Genes Dev. 14:3191-3203.

37. Rosenberger, C., et al. 2002. Expression of hypoxiainducible factor-1alpha and -2alpha in hypoxic and ischemic rat kidneys. J. Am. Soc. Nephrol. 13:1721-1732.

38. Oliver, J.A. 2004. Adult renal stem cells and renal repair. Curr. Opin. Nephrol. Hypertens. 13:17-22.

39. Maeshima, A., Yamashita, S., Nojima, Y. 2003. Identification of renal progenitor-like tubular cells that participate in the regeneration process of the kidney. J. Am. Soc. Nephrol. 14:3138-3146.

40. Sklar, A.H., et al. 1990. A population study of renal function in sickle cell anemia. Int. J. Artif. Organs. 13:231-236.

41. Powars, D.R., et al. 1991. Chronic renal failure in sickle cell disease: risk factors, clinical course, and mortality. Ann. Intern. Med. 115:614-620.

42. Falk, R.J., et al. 1992. Prevalence and pathologic features of sickle cell nephropathy and response to inhibition of angiotensin-converting enzyme. N. Engl. J. Med. 326:910-915.

43. Sanerkin, N.G. 1966. Chronic phenacetin nephropathy. (With particular reference to the relationship between renal papillary necrosis and "chronic interstitial nephritis”). Br. J. Urol. 38:361-370.

44. Kincaid-Smith, P. 1967. Pathogenesis of the renal lesion associated with the abuse of analgesics. Lancet. 1:859-862.

45. Bulger, R.E., and Siegel, F.L. 1975. Alterations of the renal papilla during mercuric chloride-induced acute tubular necrosis. Lab. Invest. 33:712-719. 